DOI: http://dx.doi.org/10.18524/1810-4215.2017.30.114381

\title{
PHOTOMETRY AND BLAZHKO EFFECT IN RR LYR-TYPE STAR AE LEO
}

\author{
S.N. Udovichenko, L.E. Keir \\ Astronomical Observatory, Odessa National University, \\ Odessa, Ukraine, udovich222@ukr.net
}

ABSTRACT. The photometric observations for the RR Lyr-type star AE Leo in the Astronomical station near Odessa using the 48-cm reflector AZT-3 and the CCD photometer equipped with the $\mathrm{V}$ filter have been carried out. The light curves shows variable amplitude of light maxima and the moderate Blazhko effect. The analysis of the O-C light maxima for our observations and the ASAS, NSVS data indicates the secular period changes.

Keywords: Stars: oscillations - stars; variables: RR Lyr-type stars: individual: AE Leo.

\section{Introduction}

AE Leo (AN 1935.0206, GSC 1437.00734, NSVS 10338360), $\left(\alpha_{J 2000.0}=11^{h} 26^{m} 12.2^{s}\right.$; $\left.\delta_{J 2000.0}=+17^{\circ} 39^{\prime} 39.7^{\prime \prime}\right)$ The variability of the star was found by Morgenroth in 1935, as a variable star in list of the 31 new variables (Morgenroth, 1935). The finding chart for this star was published by N.E.Kurochkin (1949). The star thoroughly was investigated by L.Meinunger, which determined the primary period and 32 times of light maxima, using the old photographic plates from 1913 to 1961 years (Meinunger, 1961). The visual observations in 20 century were carried out by B.N.Firmanyuk and V.G.Derevyagin in 1987, Huebscher et al., in 1989 (Paschke, 2008). Now AE Leo is known as RR Lyr-type RRab star (GCVS) with amplitude $11 .^{m} 6-12 .^{m} .8(\mathrm{P})$ and period $0 .{ }^{d} 626723$ (Samus et al., 2011).

\section{Observations}

To study the physical explanation for the Blazhko effect, we have to get as much as possible detailed observations of RR Lyr-type stars. The photometric CCD observations of AE Leo in Astronomical station near Odessa in observation season 2013,2015,2017 years have been carried out.

Two stars were chosen as comparison and check stars $\left(\operatorname{comp}=\mathrm{UCAC} 4 \quad 539-050620, \quad \mathrm{~V}\right.$ comp $=14 .^{m} 157$

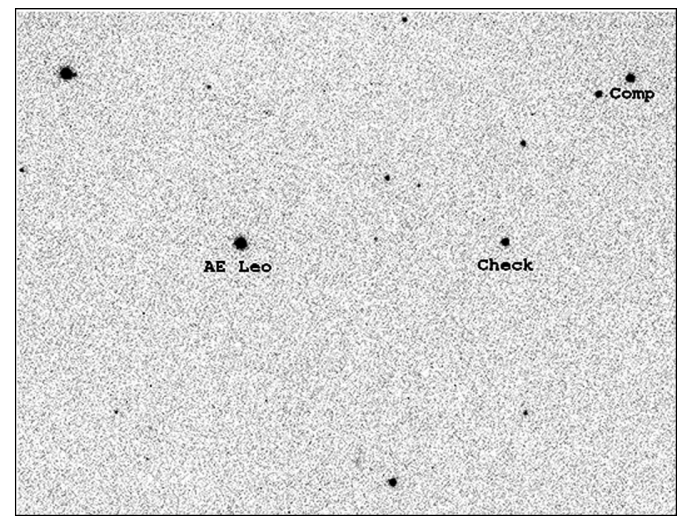

Figure 1: The finding chart AE Leo with the comparison and check stars marked.

(APASS, 2010), check=UCAC4-539-050615). The 48 cm reflector AZT-3 with the f/4.5 Newtonian focus and CCD photometer with chip Sony ICX429ALL (600x800 pixels), equipped with $\mathrm{V}$ filter, Peltier cooler were used (Udovichenko, 2012). The hermetic housing and thermoelectric (Peltier) cooler provide a temperature difference between the sensor CCD and the environment of about $-40^{\circ} \mathrm{C}$, and the temperature was supported by a constant. The exposure time for variable and comparison stars for the most part were chosen to except a saturation of frame and consist 90 sec. More then 3900 CCD frames were gathered during 28 nights.

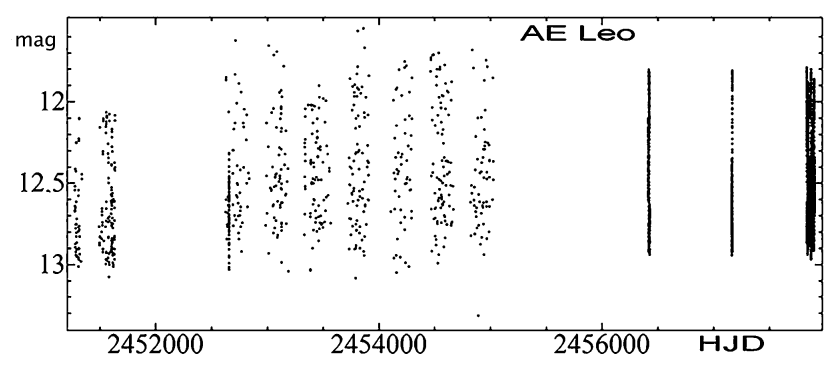

Figure 2: The all data set of observations with NSVS and ASAS data. 


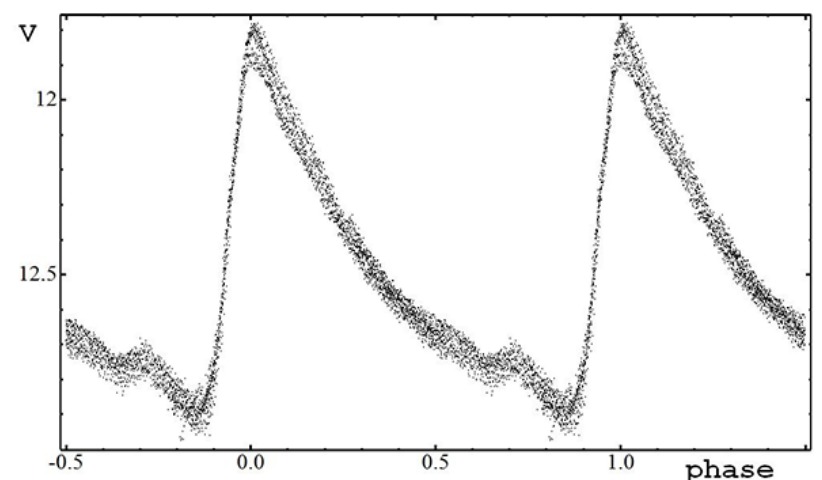

Figure 3: The phase curves of AE Leo from our observations. The moderate Blazhko modulation of amplitude reach about $0.13 \mathrm{mag}$

The standard reductions of the CCD frames were carried out using the MUNIPACK (Motl, 2009-17) software. The procedures for the aperture photometry is composed of the dark-level and flat-field corrections and determination of the instrumental magnitude and precision. To convert the differential magnitudes to the corresponding magnitudes of the variable, were used the comparison star $\mathrm{V}$ magnitude from APASScatalog (2010). The finding chart with comparison and check stars are shown in Fig. 1, the all set of observations one in Fig. 2. The errors on individual data points vary between $0.005 \mathrm{mag}$ to $0.02 \mathrm{mag}$.

\section{Frequency analysis}

For all our observations of AE Leo were determined the magnitudes comparatively of comparison star. The frequency analyses were performed using a package of computer programs with single-frequency and multiple-frequency techniques by using utilize Fourier as well as multiple-least-squares algorithms (program Period04, Lenz and Breger, 2004). The pulsation period was determined with this package as the highest peak on the Fourier amplitude spectra. The all light curves AE Leo with pulsating period are shown on fig.3. These phase curves were computed from elements:

$$
\text { MaxHJD }=2419839.549+0.6267074 * E .
$$

The value in maxima of the light curves vary from $11.77 \mathrm{mag}$ to $11.90 \mathrm{mag}$, moderate Blazhko modulation of amplitude reach about $0.13 \mathrm{mag}$ and no phase-shift modulations. The maxima of the pulsation cycles were determined for our analysis. The data of ASAS (Pojmanski, 2002) and NSVS (Wozniak, 2004) so were used for determinations of the light maxima (Fig. 4).

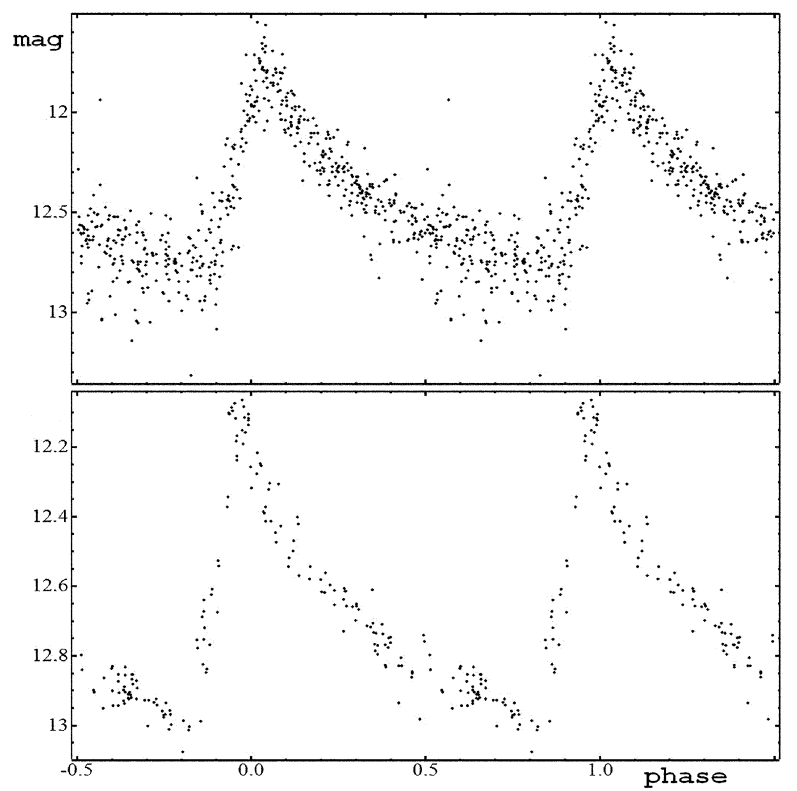

Figure 4: The phase curves of AE Leo from NSVS (bottom) and ASAS (top) data.
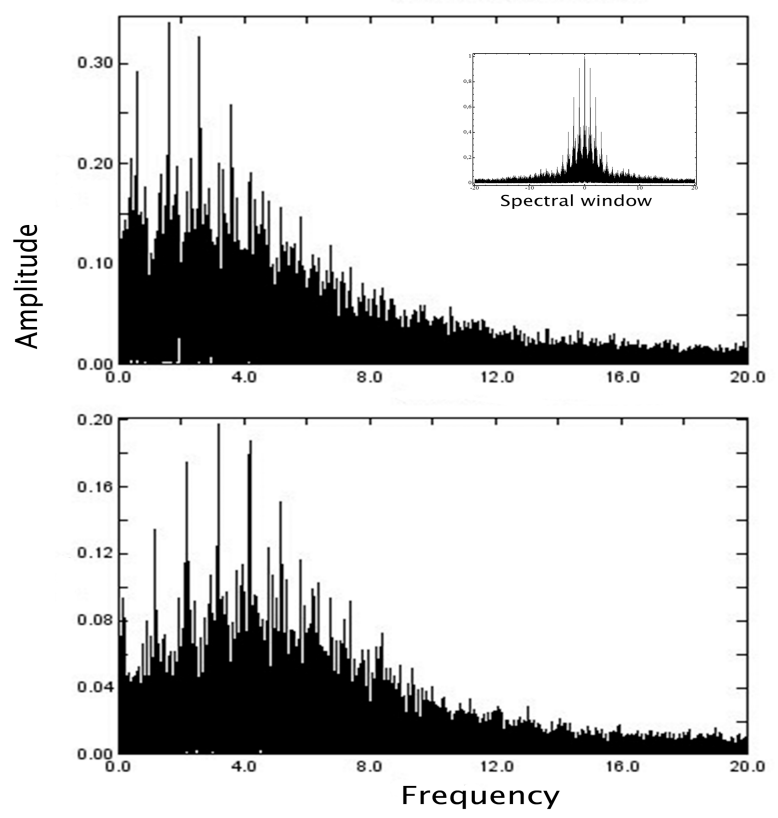

Figure 5: The Fourier amplitude spectrum of basic frequency and after prewhitening. The spectral window is shown inside first picture.

The power spectra of basic frequency and after prewhitening is shown in Fig. 5. After prewhitening of basic frequency the harmonics of basic frequency $\mathrm{kf0}$ and $f_{m}$ frequency responsible for modulation of light curves was found. The Fourier amplitude and phases of the pulsation component identified in the spectra of 


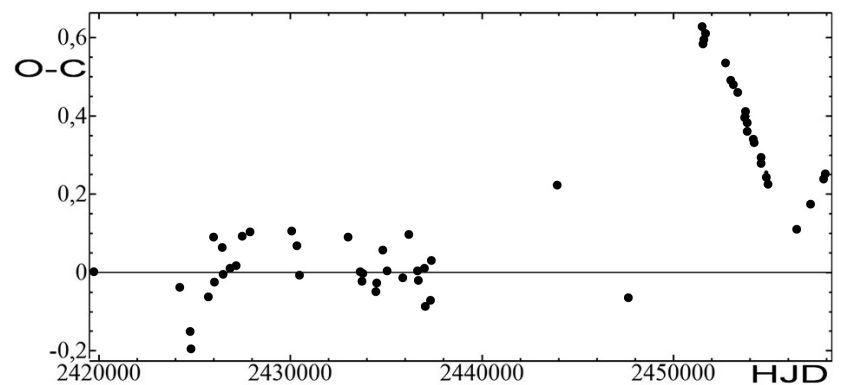

Figure 6: The mean O-C diagram of AE Leo during 100 years.

Table 1: Identified Fourier amplitude and phases of the pulsation and modulation frequencies in light curves of AE Leo.

\begin{tabular}{ccccc}
\hline Identif. & Frequency & Amplitude & Phase & S/N \\
\hline f0 & 1.595519 & 0.370 & 0.86 & 5.3 \\
2f0 & 3.191036 & 0.190 & 0.47 & 57.6 \\
3f0 & 4.786535 & 0.117 & 0.95 & 40.0 \\
4f0 & 6.380688 & 0.078 & 0.72 & 31.2 \\
5f0 & 7.977561 & 0.044 & 0.15 & 18.3 \\
6f0 & 9.573121 & 0.024 & 0.39 & 10.4 \\
7f0 & 11.16721 & 0.019 & 0.40 & 8.3 \\
f0-fm & 1.51316 & 0.015 & 0.11 & 4.6 \\
\hline
\end{tabular}

Table 2: The time of maxima AE Leo

\begin{tabular}{cc}
\hline Time HJD & Time HJD \\
\hline 2456416.467 & 2457165.440 \\
2457835.449 & 2457840.464 \\
2457847.355 & 2457872.429 \\
2457899.380 & \\
\hline
\end{tabular}

the light curves of AE Leo are presented in Table 1. The basic frequency denoted as $\mathrm{f}_{0}$, the modulation frequency of Blazhko effect denote as $f_{m}$. We find seven frequencies, but, perhaps, there are more frequencies in the pulsation spectra of AE Leo.
From obtained data (Fourier analysis and the maxima height change) the preliminary value of Blazhko effect period is amount $12^{d}$.

\section{Pulsation-period variations, the O-C diagram}

We have collected all available data from literature and WWW-resource for AE Leo maxima and constructed the updated O-C diagram, using the elements from GCVS. The resulting diagram is plotted in Fig. 6. These data indicate that AE Leo shows the secular period changes. The time of maxima from our observations, obtained by fitting pulsation light curves near the maxima, listed in Table 2.

\section{References}

APASS, Star catalog: 2010, http://www.aavso.org. Benn D.: http://www.aavso.org/vstar.

Kurochkin N.E.: 1949, Perem. Zvezdy, N6, 303.

Lenz P., Breger M.: 2004, Comm.in Asteroseismology 144, p.41.

Meinunger L.: 1961, MVS N604-605.

Morgenroth O.: 1935 Astron. Nachr., 256, 281.

Motl D.: 2009-17 http://sourceforge.net/projects/cmunipack

Paschke A., Martignoni M.: 2008 http://www.ast.obs-mip.fr/users/leborgne/dbRR/

Pojmanski G.: 2002, Acta Astronomica, 52, 397.

Samus N.N., Durlevich O.V., Kazarovets E V., Kireeva N.N., Pastukhova E.N., Zharova A.V. et al.: 2011, General Catalogue of Variable Stars (GCVS database, Version 2011Jan).

Udovichenko S.N.: 2012, Odessa Astron. Publ., 25, 32.

Wozniak, P.R., Vestrand, W.T., Akerlof, C.W. et al.: 2004, Astron. J., 127, 2436. 